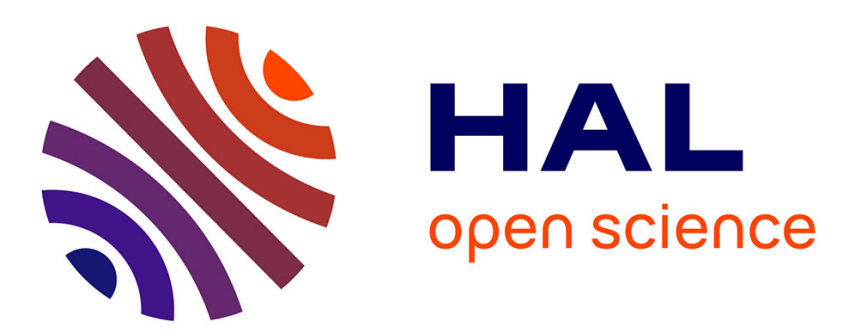

\title{
Series expansion of electrostatic potential radiated by a point source in isotropic Maxwellian plasma
}

Christian Béghin

\section{To cite this version:}

Christian Béghin. Series expansion of electrostatic potential radiated by a point source in isotropic Maxwellian plasma. Radio Science, 1995, 30 (2), pp.307-322. 10.1029/94RS03167 . insu-03191896

\section{HAL Id: insu-03191896 \\ https://hal-insu.archives-ouvertes.fr/insu-03191896}

Submitted on 9 Apr 2021

HAL is a multi-disciplinary open access archive for the deposit and dissemination of scientific research documents, whether they are published or not. The documents may come from teaching and research institutions in France or abroad, or from public or private research centers.
L'archive ouverte pluridisciplinaire HAL, est destinée au dépôt et à la diffusion de documents scientifiques de niveau recherche, publiés ou non, émanant des établissements d'enseignement et de recherche français ou étrangers, des laboratoires publics ou privés. 


\title{
Series expansion of electrostatic potential radiated by a point source in isotropic Maxwellian plasma
}

\author{
C. Béghin \\ Laboratoire de Physique et Chimie de l'Environnement, CNRS, Orléans, France
}

\begin{abstract}
A new algebraic approach is proposed to calculate the electrostatic potential distributed around a point source in isotropic Maxwellian plasma. The method derives a power series expansion of the radial distance from the source with frequency-dependent coefficients. Distance and frequency are normalized to the Debye length and to the plasma frequency, respectively, so that the expression keeps its entire generality whatever the experimental conditions might be. The proposed method is based upon the Mittag-Lefler expansion of the inverse of the plasma dispersion function for the infinite series of Landau poles. After mathematical clarification of the validity of this expansion, a significant correction of the previous works leads to a self-consistent interpretation of the true contribution of the higher-order poles at large distance from the source. The power series expansion is compared to the classical so-called "Landau wave approximation" which is proved to include in reality the contribution of higher-order poles independently from the plasma temperature. For practical use the power expansion is needed to obtain a precise result at distances from the source shorter than about 15 Debye lengths, while the Landau wave approximation gives correct results at larger distances. This work provides all necessary baselines for precise three-dimensional modeling of mutual impedance devices to be used in space plasma experiments where the Debye length is comparable to the spacecraft size.
\end{abstract}

\section{Introduction}

Since the historical paper of Landau [1946], the expression of electric perturbations in terms of potential and field induced in a Maxwellian plasma by pulsating charges has been studied extensively by innumerable authors, and under many different experimental conditions. Basics of such a classical problem are quite simple, but the algebraic difficulties in deriving a result easily usable in real applications are the cause of this large amount of literature. The main difficulty comes from the fact that the plasma dispersion function has an infinite number of roots for a Maxwellian electron distribution, the contribution of which has to be considered in some conditions, in addition to that of Landau's dominant pole [see Derfler

Copyright 1995 by the American Geophysical Union.

Paper number 94RS03167.

0048-6604/95/94RS-03167\$08.00 and Simonen, 1969]. Consequently, people have considered different kinds of approximations adapted to each experimental conditions, what is satisfactory when first-order estimates are sufficient. But when the purpose is to use the system response as a plasma diagnostic, which is the case of mutual impedance probes as initially proposed by Storey et al. [1969], a more rigorous theoretical treatment becomes necessary.

The simplest approximations first concerned the thermal distribution itself, starting from the water-bag distribution [see Grard, 1969] leading one to consider only one single real pole, that is, the adiabatic Langmuir wave solution propagating without any damping at frequencies greater than the plasma frequency $\omega_{p}$. Then, the multiple water-bag model [Navet and Bertrand, 1971] and the Cauchy distribution [Rooy et al., 1972] were proposed, both leading to a finite number of roots of the plasma dispersion equation and to the ability to approximate 
asymptotically the Maxwellian distribution. One of the most extensive investigations regarding the relative contribution between the predominating Landau's pole and all other damped poles was made by Simonen [1966] and Derfler and Simonen [1969], while exact solutions were obtained by direct numerical integration [Buckley, 1968], allowing a good estimate of the validity of approximations.

The results from those works have been used for interpretation of most space experiments where the mutual impedance devices developed by the Orléans group were flown for plasma diagnostics, as well as on board rockets [Béghin, 1971; Béghin and Debrie, 1972; Chasseriaux et al., 1972; Pottelette et al., 1981], and on board satellites [Décréau et al.,1978; Béghin et al., 1982; Décréau et al., 1991]. Most of these experiments used a large array of antennas compared to the ambient Debye length and the probes were installed at the edges of long booms, so that conventional approximations were satisfactory. But, due to constraints appearing with space vehicles on which long booms may be difficult to be implemented, mutual impedance devices must work now even for short distances and must take account of the presence of spacecraft body. Then, a precise modeling of the environment and mapping of potential induced by the current source antennas becomes necessary. The aim of this paper is to provide the baseline for general applications, for elementary point sources immersed in an isotropic Maxwellian plasma, that is, when the steady magnetic field can be neglected, according to the condition $\omega_{c} \ll \omega_{p}$, where $\omega_{c}$ is the electron cyclotron frequency.

In the following we first summarize the basics for calculation of the potential radiated by a point source, using reference works and adding some necessary correction of mathematical nature to the expansion of the inverse of the longitudinal dielectric constant which was originally proposed by Derfler [1966]. Then we show that the potential can be expressed in the form of a power series expansion of the normalized radial distance to the source, with frequency-dependent coefficients. After that the main point is to determine the analytical expression of these coefficients. Results of the exact power expansion are compared with the conventional Landau pole approximation for several frequencies around $\omega_{p}$ as a function of the distance. This will confirm the previous result [Chasseriaux et al., 1972] that this approximation is satisfactory for distances larger than $\sim 15 \lambda_{D}$, where $\lambda_{D}$ is the Debye length. Finally, one example of mutual impedance response is given, corresponding to the experimental conditions expected to be encountered with the probe to be installed on board the forthcoming MARS 96 spacecraft.

\section{Landau Poles Expansion}

The potential $\phi$ induced by a pulsating point charge $Q \exp (i \omega t)$ at a radial distance $r$ in an isotropic plasma is given by [see Chasseriaux et al., 1972]

$$
\phi=\frac{Q}{4 \pi \varepsilon_{0}} \frac{2}{\pi} \lim _{\lim \rightarrow 0}^{0} \int_{-\infty}^{\sin k r} \frac{d k}{\varepsilon_{l}(k, \omega)}
$$

Here $\varepsilon_{0}$ is the free-space permittivity, $k$ the wavenumber, and $\varepsilon_{l}(k, \omega)$ the longitudinal dielectric constant. In the following, except when specified, we consider $\omega$ to be purely real, having in mind that it is the limit reached when its small negative imaginary part tends to zero, corresponding to the steady state of the source after a transient growing phase. For a collisionless isotropic Maxwellian plasma, this dielectric constant is given by

$$
\varepsilon_{l}(k, \omega)=1-\frac{\omega_{p}^{2}}{k^{2} v_{t}^{2}} Z^{\prime}\left(\frac{-\omega}{|k| v_{t}}\right)
$$

where $v_{t}$ is the thermal velocity defined by $v_{t}^{2}=2 \kappa T_{c} / m, \mathrm{k}$ is the Boltzmann constant, $T_{c}$ and $m$ the electron temperature and mass respectively, and $Z^{\prime}(z)$ the first derivative of the well-known plasma dispersion function $Z(z)$ [Fried and Conte, 1961], the definition of which is

$$
Z(z)=\frac{1}{\sqrt{\pi}} \int_{-\infty}^{\infty} \frac{e^{x^{2}}}{x-z} d x \quad(\operatorname{Im} z>0)
$$

with an analytic continuation for $\operatorname{Im} z \leq 0$.

It must be mentioned here that the linear plasma wave description is used in this theory, so that the pulsating point source is supposed to have an amplitude small enough to not disturb the surrounding plasma. This condition could be not applicable to experiments such as topside or relaxation sounders, where high-level voltages are used to feed the antenna. 
Then, for a given antenna device, it is necessary to determine the limit of the linear theory as a function of the local plasma parameters (electron density and Debye length). The condition to be satisfied for linearity is that the electric energy per volume unit in the region under consideration around the source (computed using a linear theory such as that presented here) must be lower than the thermal energy density of the plasma. This condition is usually satisfied in mutual impedance devices even near the sources.

One can easily see that the integrant of (1) exhibits in the entire complex plan of $k$ as many poles as the number of roots of the dispersion equation $\varepsilon_{t}=0$. Then for analytic evaluation of the integral in (1) it is convenient to express the term $1 / \varepsilon_{l}$ by its expansion in a Mittag-Lefler series, with respect to all poles, as initially proposed by Derfler [1966]. At this stage it is necessary to rewrite (2) for complex variables, using the symmetry properties of the dispersion equation and analytic continuation of the plasma dispersion function [Derfler and Simonen, 1969]. First, let us introduce the following dimensionless variables

$$
K=k \lambda_{D} ; \mathbf{Q}=\frac{\omega}{\omega_{p}} ; z=\frac{\Omega}{\sqrt{2} K} ; \rho=\frac{r}{\lambda_{D}}
$$

where $\lambda_{D}$ is the Debye length defined as

$$
\lambda_{D}=\frac{\sqrt{\kappa T_{e} / m}}{\omega_{p}}=\frac{\nu_{t}}{\sqrt{2} \omega_{p}}
$$

Then the term $1 / \varepsilon_{l}$ becomes

$$
\begin{gathered}
\frac{1}{\varepsilon_{l}(K, \Omega)}=\frac{1}{1-\frac{Z^{\prime}(\Omega / \sqrt{2} K)}{2 K^{2}}} \\
=\frac{\Omega^{2}}{\Omega^{2}-z^{2} Z^{\prime}(z)}
\end{gathered}
$$

Note our dimensionless $K$ is $\sqrt{2}$ smaller than that used by Derfler and Simonen [1969], due to our choice to normalize the wavenumber with respect to $\lambda_{D}$ instead of the thermal velocity. The poles of (6) are the wellknown infinite series of Landau poles, which will be considered here for complex $K$ and real $\Omega$, or more precisely for $\Omega-i v$ when $v \rightarrow 0$.
The behavior of these poles in the complex plane, when $\Omega$ varies from 0 to above 1 , is summarized in Figures 1 through 3 . Using the usual notations, the dominating Landau pole $K_{1}$ exists only for $\Omega \geq 1$, lying in the upper left-hand quarter of the complex plane (Figure 1). The purely imaginary poles $K_{2}$ and $K_{-2}$ (for $\Omega<1$ ) are plotted in Figure 2, and the higher-order poles $K_{n}$ in Figure 3, knowing that each pole $K_{n}$ is associated with its opposite conjugate according to the relationship $K_{-n}=-K_{n}^{*}$.

The function $1 / \varepsilon_{1}$ can be expanded in form of an infinite sum of the principal parts at all poles of the complex plane (Mittag-Leffler series) under certain conditions [see Jeffreys and Swirles, 1966]. The most important condition in our case is that the function must be analytic at the origin. Thus this cannot be strictly applicable with the variable $K$, because when $K \rightarrow 0$ an infinity of poles $K_{n}$ and $K_{-n}$ crowd toward zero along the rays arg $(K)=3 \pi / 4$ (Figure 3 ) and $\pi / 4$ respectively. Indeed, the Mittag-Leffler expansion, for a given function $f(z)$ is

$$
f(z)=f(0)+\sum_{|n|}^{\infty}\left(\frac{b_{n}}{z_{n}}+\frac{b_{n}}{z-z_{n}}\right)
$$

where $b_{n}$ is the residue at the pole $z_{n}$ and the sum is taken from $n=1, \pm 2, \ldots, \pm \infty$.

Instead of the variable $K$, let us consider $z$, as defined in (4). Now, for any given $\Omega$, one can check in the last term of (6) that $1 / \varepsilon_{l}$ is analytic at $z=0$ since $Z$ is continuously derivable in that area which corresponds to a pole-free region in $K$ space when $K \rightarrow \infty$. Obviously, here $f(0)=1$.

Using the properties of the function $Z$ and its derivatives, the residue is given by

$$
\begin{aligned}
b_{n}= & \frac{1}{\left(\frac{d \varepsilon_{l}}{d z}\right)_{z=z_{n}}}=\frac{z_{n}}{2 z_{n}^{2} \varepsilon_{c}-3} \\
& \equiv \frac{\Omega}{\sqrt{2}} \frac{K_{n}}{\Omega^{2}-1-3 K_{n}^{2}}
\end{aligned}
$$

where $\varepsilon_{c}$ is the usual cold plasma dielectric constant given by $\varepsilon_{c}=1-\Omega^{-2}$.

Using (7) and (8), $1 / \varepsilon_{l}$ can be now expressed indifferently in function of either $z$ or $K$ 


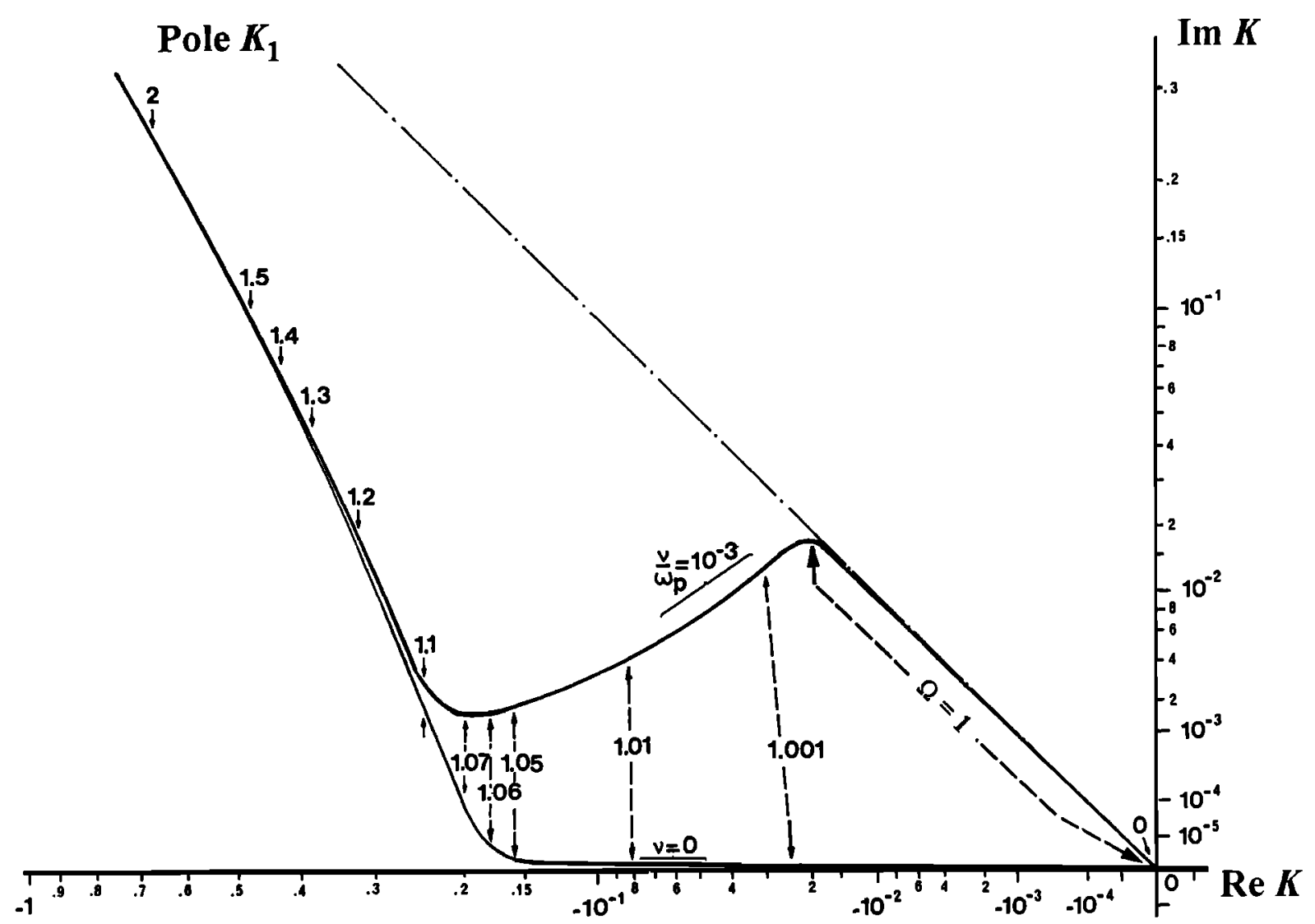

Figure 1. Loci of pole $K_{1}$ in the complex plane as a function of the frequency (indicated by arrows) for real $\Omega(v=0)$, and with a small imaginary part in order to understand the limit when $v \rightarrow 0$. Note the unusual power-law scale which offers some advantage of the log scale with the possibility to cross the origin from positive to negative values.

$$
\begin{gathered}
\frac{1}{\varepsilon_{l}}=1+\sum_{|n|}^{\infty} \frac{b_{n}}{z_{n}}+\sum_{|n|}^{\infty} \frac{b_{n}}{z-z_{n}} \equiv 1+ \\
\sum_{|n|}^{\infty} \frac{K_{n}^{2}}{\Omega^{2}-1-3 K_{n}^{2}}-\sum_{|n|}^{\infty} \frac{K_{n}^{2}}{\Omega^{2}-1-3 K_{n}^{2}} \frac{K}{K-K_{n}}
\end{gathered}
$$

which could be considered as the Mittag-Leffler expansion versus the variable $K$ as well, with the significant difference that now the two first constant terms (independent from $K$ ) are definite, since there is no reference to the undetermined value at the singular point $K=0$. To calculate the value of this constant, either we compute directly the infinite sum for given
$\Omega$, or better, we can derive an analytic expression. The result of the numerical computation is shown in Figure 4 , for $\Omega=1.01$. The asymptotic behavior of the sum indicates clearly that

$$
1+\sum_{|n|}^{\infty} \frac{b_{n}}{z_{n}}=\frac{3}{4 \varepsilon_{c}}
$$

while the direct Mittag-Leffler expansion with respect to $K$ gives $1 / \varepsilon_{c}$, the value attributed in (6) when $K=0$ [Chasseriaux et al., 1972]. For the analytic demonstration of $(10)$, let us consider the expansion of $1 / \varepsilon_{l}$ versus real $z$ given by (9) when $z \rightarrow \pm \infty$. From (6), knowing that $z^{2} Z^{\prime}(z)$ tends asymptotically to 1 , we get $1 / \varepsilon_{l} \rightarrow 1 / \varepsilon_{c}$. Thus we can write 


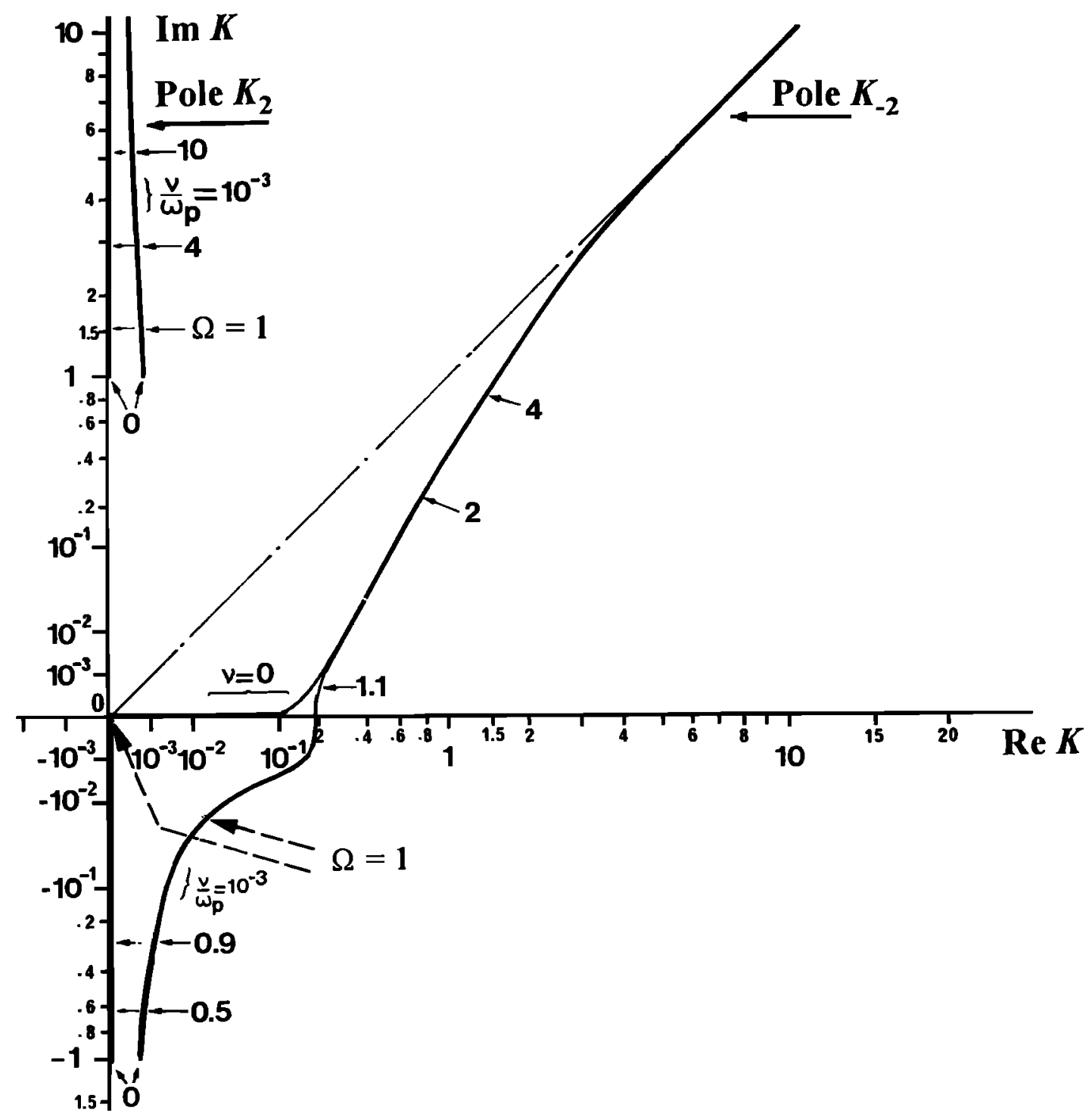

Figure 2. Loci of poles $K_{2}$ and $K_{-2}$, in the same manner as those in Figure 1.

$$
1+\sum_{|n|}^{\infty} \frac{b_{n}}{z_{n}}=\frac{1}{\varepsilon_{c}}-\lim _{z \rightarrow \infty} \sum_{|n|}^{\infty} \frac{b_{n}}{z-z_{n}}
$$

This equation shows why the Mittag-Leffler expansion in $K$ is indefinite, as long as the way to approach zero is not determined. Indeed, the second term of the right-hand side of (11) is not zero, as could be thought at first glance. To calculate this term, we will remark that the contribution of the first finiteorder Landau poles (finite values of $z_{n}$ ) can be neglected while $z$ goes to infinity. Then, the asymptotic approximation of higher-order poles when $|n|$ tends to infinity [Derfler and Simonen, 1969] can be used

$$
\operatorname{Lim}_{|n| \rightarrow \infty} z_{n}=-\sqrt{|n| \pi}[\operatorname{sgn}(n)+i]
$$

and the discrete sum can be transformed to integral, putting $\pi n=x$, with $x$ varying from 0 to infinity. After some algebra we found 


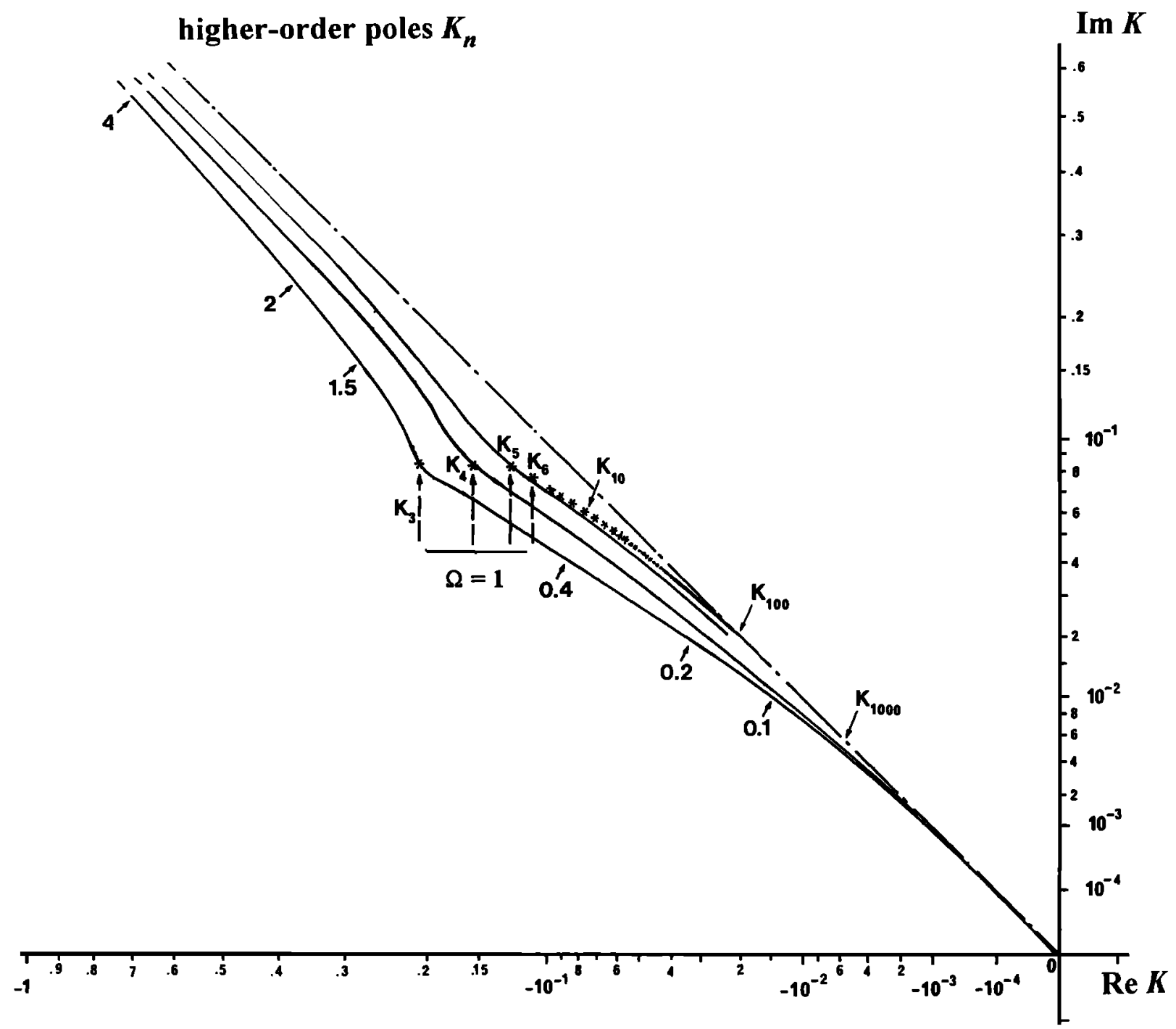

Figure 3. Loci of poles $K_{n}$ for $v=0$, knowing that the same family exists symmetrically for $K_{-n}$ to the imaginary axis.

$$
\begin{aligned}
& \operatorname{Re}\left(\begin{array}{l}
\operatorname{Lim} z \rightarrow \infty \\
z \rightarrow n \mid
\end{array} \sum_{|n|}^{\infty} \frac{b_{n}}{z-z_{n}}=\frac{24+16 \varepsilon_{c} z^{2}}{\pi}\right. \\
& \int_{0}^{\infty} \frac{x^{2} d x}{\left(9+16 x^{2} \varepsilon_{c}^{2}\right)\left(z^{4}+4 x^{2}\right)}=\frac{1}{4 \varepsilon_{c}}
\end{aligned}
$$

Similar calculation proves that the imaginary part is zero, completing the demonstration of (10).

The integrant in (1) is now well-defined everywhere in the complex plane. Then, changing $k$ into $-k$ in the integral and using the dimensionless variables, we rewrite ( 1 ) in its final form

$$
\begin{aligned}
\phi=\frac{Q}{4 \pi \varepsilon_{0} r} & {\left[\frac{3}{4 \varepsilon_{c}}+\frac{2}{\pi} \sum_{|n|}^{-} \frac{K_{n}^{2}}{1-\Omega^{2}+3 K_{n}^{2}}\right.} \\
& \left.\cdot \int_{0}^{-} \frac{\sin x d x}{x+K_{n} p}\right]
\end{aligned}
$$

which differs from the expression derived by 


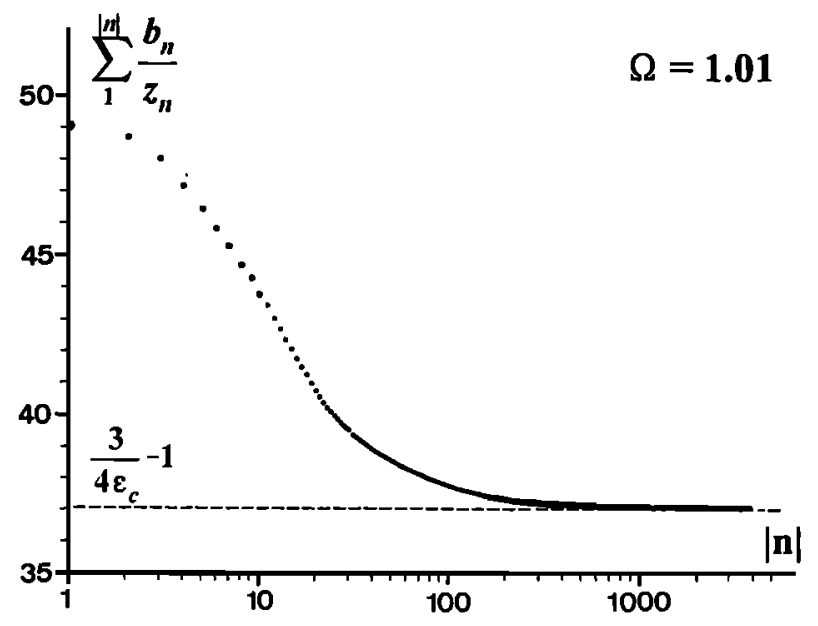

Figure 4. Computed sum of the excitation coefficients of Landau poles versus the order $|n|$, for $\Omega=1.01$.

Chasseriaux et al. [1972] by the term $3 / 4 \varepsilon_{c}$ instead of $1 / \varepsilon_{c}$.

\section{Series Expansion of the Potential}

The integral in (14) is an auxiliary function of sine and cosine integrals [Abramowitz and Stegun, 1972] defined as

$$
\begin{gathered}
f\left(K_{n} p\right)=\int_{0}^{-} \frac{\sin x d x}{x+K_{n} p} \\
=C i\left(K_{n} p\right) \sin \left(K_{n} p\right)-s i\left(K_{n} p\right) \cos \left(K_{n} p\right)
\end{gathered}
$$

It is a multivaluated function, as a cosine integral is, with the branch cut along the real negative axis, where there is no pole, according to the discussion in previous section. Using the series expansions of each individual function of $(15)$, we obtain

$$
\begin{aligned}
& f\left(K_{n} p\right)=\frac{\pi}{2}\left[1+\sum_{q=1}^{\infty}(-1)^{q} \frac{p^{2 q}}{(2 q) !} K_{n}^{2 q}\right]+ \\
& \sum_{q=0}^{-}(-1)^{q}\left(\gamma+\ln K_{n} p-\sum_{p=1}^{2 q+1} \frac{1}{p}\right) \frac{p^{2 q+1}}{(2 q+1) !} K_{n}^{2 q+1}
\end{aligned}
$$

with the determination $\left|\arg \left(K_{n} \rho\right)\right|<\pi$, and where $\gamma$ is the Euler's constant $\gamma=0.5772156649$....

Then, substituting this expression to the integral in (14), the potential is expressed on form of a series expansion versus the variable $\rho$, with coefficients (infinite sums over $K_{n}$ ) depending only on the frequency, plus a constant which is easily found to be 1 , using (8) and (10). We get three different kinds of coefficients

$$
\begin{gathered}
S_{2 q}=\sum_{|n|}^{\infty} \frac{K_{n}^{2 q+2}}{1-\Omega^{2}+3 K_{n}^{2}} ; S_{2 q+1}=\sum_{|n|}^{\infty} \frac{K_{n}^{2 q+3}}{1-\Omega^{2}+3 K_{n}^{2}} \\
L_{2 q}=\sum_{|n|}^{\infty} \frac{K_{n}^{2 q+3} \ln K_{n}}{1-\Omega^{2}+3 K_{n}^{2}}
\end{gathered}
$$

From considerations about the symmetry of poles, that is, $K_{1}=-K_{-2}{ }^{*}$ when $\Omega>1, K_{n}=-K_{-n}{ }^{*}$ for $n>2$, and knowing that $K_{2}$ and $K_{-2}(\Omega<1)$ are purely imaginary, we deduce that the even coefficients $S_{2 q}$ are purely real, and the odd ones are purely imaginary, while the coefficients $L_{2 q}$ have both real and imaginary parts due to $\ln K_{n}$. Then, the real and imaginary parts of (14) are

$$
\begin{gathered}
\operatorname{Re}\left(\phi / \phi_{O}\right)=1+\sum_{q=1}^{\infty}(-1)^{q}\left[\frac{p^{2 q}}{(2 q) !} S_{2 q}-\right. \\
\left.\frac{\rho^{2 q-1}}{(2 q-1) !} \frac{2}{\pi} \operatorname{Re}\left(L_{2 q-2}\right)\right] \\
\operatorname{Im}\left(\phi / \phi_{O}\right)=\frac{2}{\pi} \sum_{q=0}^{\infty}(-1)^{q} \frac{p^{2 q+1}}{(2 q+1) !} \\
{\left[\left(\gamma+\ln p-\sum_{p=1}^{2 q+1} \frac{1}{p}\right) S_{2 q+1}+\operatorname{Im}\left(L_{2 q}\right)\right]}
\end{gathered}
$$

respectively, where $\phi_{0}$ is the free-space potential, $\phi_{0}=Q / 4 \pi \varepsilon_{0} r$.

This is the desired series expansion of the potential versus the distance from the source with frequency dependent coefficients. One can see that the potential tends to its vacuum value when $\rho$ tends to zero. The next point now is to determine the coefficients.

\section{Calculation of the Coefficients $S$ and $L$}

For practical applications the coefficients given by (17) can be computed and tabulated once and for all for every desired normalized frequency. Here we propose to determine them analytically for better understanding of their behavior versus the frequency. 
The easier coefficients to determine are the coefficients $S$. The first one, $S_{0}$, is already known, as given from (10), that is, $S_{0}=1-3 / 4 \varepsilon_{c}$. The following terms are obtained by successive derivation of the dispersion equation. Using (6) and (9), we can write

$$
\begin{aligned}
& \left.\frac{\partial^{p} \varepsilon_{z}}{\partial z^{p}}\right|_{z=0}=\left.\frac{\partial^{p}}{\partial z^{p}}\left[1-\frac{z^{2} Z^{\prime}(z)}{\Omega^{2}}\right]\right|_{z=0} \\
& =\left.\frac{\partial^{p}}{\partial z^{p}}\left[1-\varepsilon_{l} \sum_{|n|}^{\infty} \frac{b_{n}}{z_{n}} \frac{z}{z-z_{n}}\right]\right|_{z=0}
\end{aligned}
$$

For $p=1$ to 5 , one finds from (19) after some algebra

$$
\begin{aligned}
& \sum_{|n|}^{\infty} \frac{b_{n}}{z_{n}^{2}}=0 ; \sum_{|n|}^{\infty} \frac{b_{n}}{z_{n}^{3}}=\frac{2}{\Omega^{2}} ; \sum_{|n|}^{\infty} \frac{b_{n}}{z_{n}^{4}}=i \frac{2 \sqrt{\pi}}{\Omega^{2}} \\
& \sum_{|n|}^{\infty} \frac{b_{n}}{z_{n}^{5}}=-\frac{4}{\Omega^{2}}\left(1+\frac{1}{\Omega^{2}}\right) ; \sum_{|n|}^{\infty} \frac{b_{n}}{z_{n}^{6}}=-i \frac{2 \sqrt{\pi}}{\Omega^{2}}\left(1+\frac{4}{\Omega^{2}}\right)
\end{aligned}
$$

Then, from (8) and (20) we obtain successively

$$
\begin{gathered}
S_{p}=-\frac{Q^{p}}{2^{p / 2}} \sum_{|x|}^{\infty} \frac{b_{n}}{z_{n}^{p+1}} \\
S_{1}=0 ; S_{2}=-1 ; S_{3}=-i \sqrt{\frac{\pi}{2}} \Omega \\
S_{4}=1+Q^{2} ; \quad S_{5}=i \sqrt{\frac{\pi}{2}} Q\left(2+\frac{Q^{2}}{2}\right)
\end{gathered}
$$

A recurrent expression for higher orders than $S_{5}$ can be obtained from (19) and (20). For that we must use the recurrent properties of the derivatives of the function $Z$ and apply Leibniz's theorem to (19). Using the following relations

$$
\left.\frac{d^{2 q+1} Z}{d z^{2 q+1}}\right|_{z=0}=(-1)^{g+1} 2^{2 q+1} q !
$$

$$
\begin{aligned}
& \left.\frac{d^{2 q} Z}{d z^{2 q}}\right|_{z=0}=i(-1)^{q} 2^{q} 1.3 .5 \ldots(2 q-1) \sqrt{\pi} \\
& \left.\frac{d^{p} \varepsilon_{l}}{d z^{p}}\right|_{z=0}=-\left.\frac{1}{\Omega^{2}} p(p-1) \frac{d^{p-1} Z}{d z^{p-1}}\right|_{z=0}
\end{aligned}
$$

we obtain the desired recurrent relationship between even coefficients, for $q \geq 3$

$$
\begin{gathered}
S_{2 q}=\frac{(-1)^{q} \Omega^{2 q-2}}{1.3 .5 \ldots(2 q-3)}\left[1-\frac{2 q-3}{\Omega^{2}} S_{2}+\ldots+\right. \\
\left.(-1)^{q+1} \frac{1.3 .5 \ldots(2 q-3)}{\Omega^{2 q-2}} S_{2 q-2}\right]+ \\
i \sqrt{\frac{\pi}{2} \frac{(-1)^{q} \Omega^{2 q-5}}{2^{q-3}(q-3) !}\left[S_{3}-\frac{2(q-3)}{\Omega^{2}} S_{5}+\ldots+\right.} \\
\left.(-1)^{q+1} \frac{2^{q-3}(q-3) !}{\Omega^{2 q-6}} S_{2 q-3}\right]
\end{gathered}
$$

and a similar one for odd coefficients, which reads

$$
\begin{gathered}
S_{2 q+1}=i \sqrt{\frac{\pi}{2}} \frac{(-1)^{q} \Omega^{2 q-1}}{2^{q-1}(q-1) !}\left[1-\frac{2(q-1)}{\Omega^{2}} S_{2}+\ldots+\right. \\
\left.(-1)^{q+1} \frac{2^{q-1}(q-1) !}{\Omega^{2 q-2}} S_{2 q-2}\right]- \\
\frac{(-1)^{q} \Omega^{2 q-4}}{1.3 .5 \ldots(2 q-5)}\left[S_{3}-\frac{2 q-5}{\Omega^{2}} S_{5}+\ldots+\right. \\
\left.(-1)^{q} \frac{1.3 .5 \ldots(2 q-5)}{\Omega^{2 q-4}} S_{2 q-1}\right]
\end{gathered}
$$

One can see from (21), (23), and (24) that each $S_{p}(\Omega)$ is a polynomial of degree $p-2$ for $\Omega$, with all its coefficients of the same sign but alternate with respect 
to $S_{p+1}$. For $\Omega \rightarrow 0$ we obtain the limits $S_{2 q}(0)=(-1)^{q}$ and $S_{2 q+1}(0)=0$. Moreover, all $S_{p}$ 's are finite at $\Omega=1$.

The coefficients $L_{2 q}$ are more difficult to calculate analytically due to the presence of logarithm of $K_{n}$ in (17). However, the real part is quite easy to obtain, using the symmetry properties of the poles $K_{n}$. Indeed, for $n \geq 3$ and with the determination $\left|\arg K_{ \pm n}\right|<\pi$, we have

$$
\begin{gathered}
\operatorname{Re}\left(\ln K_{n}\right)=\operatorname{Re}\left(\ln K_{-n}\right)=\ln \left|K_{n}\right| \\
\quad \operatorname{Im}\left(\ln K_{n}\right)=i \pi-\operatorname{Im}\left(\ln K_{-n}\right)
\end{gathered}
$$

and for $\Omega>1$ the same relationship exists between the poles $K_{1}$ and $K_{-2}$. Then, the contribution of the purely imaginary pole $K_{2}$ being simply $\ln K_{2}=\ln \left|K_{2}\right|+i \pi / 2$, we get from (17)

$$
\operatorname{Re}\left(L_{2 q}\right)=i \frac{\pi}{2} S_{2 q+1} \quad(\Omega>1)
$$

For $\Omega<1$ the contribution of $K_{-2}$ added to that of other poles leads one to write $\ln K_{-2}=\ln \left|K_{-2}\right|+i \pi / 2-i \pi$. Then, we get

$$
\begin{aligned}
& \operatorname{Re}\left(L_{2 q}\right)=i \frac{\pi}{2} S_{2 q+1}- \\
& i \pi \frac{K_{-2}^{2 q+3}}{1-\Omega^{2}+3 K_{-2}^{2}} \quad(\Omega<1)
\end{aligned}
$$

This result shows a discontinuity at $\Omega=1$ which needs some attention. First, let us estimate the limit of the second term in the right-hand side of (27) when $\Omega \rightarrow 1$. The solution of the dispersion equation $\varepsilon_{l}(K, \Omega)=0$ for the imaginary pole $K_{-2}$, expressed as a power expansion for the cold plasma dielectric constant $\varepsilon_{o}$ using the asymptotic expansion of $Z^{\prime}(z)$, reads

$$
3 K_{-2}^{2}=\varepsilon_{c}\left[1+\frac{\varepsilon_{c}}{3}-\frac{\varepsilon_{c}^{2}}{3}+O\left(\varepsilon_{c}^{3}\right)\right]
$$

where $\varepsilon_{c}$ tends to zero by negative value when $\Omega \rightarrow 1$. Substituting in (27) the value of $K_{-2}$ given by (28), we obtain

$$
\begin{gathered}
\operatorname{Re}\left(L_{2 q}\right) \propto i \frac{\pi}{2} S_{2 q+1}- \\
\frac{\pi}{2}(-1)^{q} \frac{\mid \varepsilon d^{q-1 / 2}}{3^{q+1 / 2}}
\end{gathered}
$$

It is clear from (29) and (26) that the continuity exists at $\Omega=1$, that is, when $\varepsilon_{c}=0$, for all orders $q$ except for the first one, $q=0$, in which case $\operatorname{Re}\left(L_{0}\right)$ tends to - $\infty$ when $\Omega$ approaches 1 by lower values. On the other hand, since $S_{1}=0$ after (21), we get $\operatorname{Re}\left(L_{0}\right)=0$ for $\Omega>1$. This discontinuity at $\Omega=1$ is consistent with the usual behavior of a single chargeinduced potential, which exhibits a singular point at $\omega_{p}$ [see Rooy et al., 1972]. Since the singularity affects only the coefficient $L_{0}$ associated with the first term of the power expansion for $\rho$ of the dimensionless potential, given by (18), we see immediately that the resulting contribution to the actual potential $\phi(r)$ is a constant independent of the distance $r$. Thus, for any configuration which must always satisfy the charge neutrality of the source $\left(\Sigma Q_{+}=\Sigma Q\right.$ ), this constant disappears, as the singularity at $\omega_{p}$ does. In the following we make the choice for the determination of $\operatorname{Re}\left(L_{0}\right)$ at $\Omega=1$ to be the same as for $\Omega>1$, that is, $\operatorname{Re}\left(L_{0}\right)=0$.

The last coefficient to be determined now is $\operatorname{Im}\left(L_{2 q}\right)$. The behavior of these coefficients versus the frequency, as obtained by direct computation of the summation in (17) over a quasi-infinite number of poles, is shown in Figure 5 for the first ones of the series. We notice that $\operatorname{Im}\left(L_{0}\right)$ presents a discontinuity at $\Omega=1$ like the real part, growing to infinity when $\Omega$ tends to 1 from upper values. The consequence on the potential is the same, since $\operatorname{Im}\left(L_{0}\right)$ corresponds also to the first order of $\rho$ in the imaginary part of the power

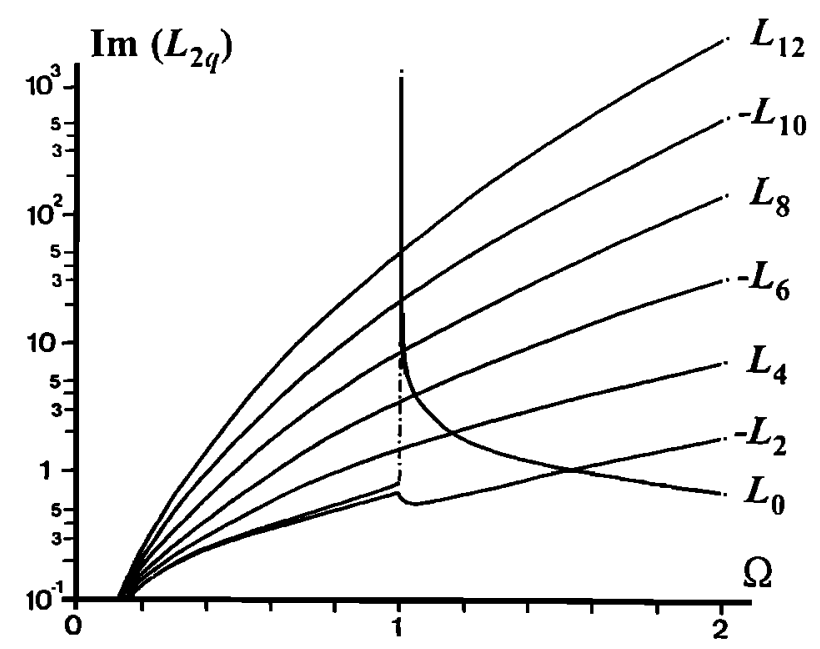

Figure 5. Plot of the imaginary part of the first orders of $L_{2 q}$ versus the frequency. 
expansion. Here we choose the determination at $\Omega=1$, which corresponds to the finite limit when $\Omega$ tends to 1 from lower values, so that, all other coefficients being finite for $\Omega=1$, both real and imaginary parts of the potential are finite at $\omega_{p}$.

There is no simple analytic expression for $\operatorname{Im}\left(L_{2 q}\right)$. However, as shown in the Appendix, one can find a generating function for the first one of the series $\left(L_{0}\right)$ and a recurrent differential equation allowing in principle to calculate the others. But for practical reasons it is more efficient and more accurate to compute and tabulate once and forever the coefficients $S$ and $L$ for a limited number of frequencies. Then, for the following we retain that this tabulation is performed using the analytic expressions for the coefficients $S_{p}$ and $\operatorname{Re}\left(L_{2 q}\right)$ and direct computation for $\operatorname{Im}\left(L_{2 q}\right)$.

The computed values of the normalized potential, using the series (18), are plotted in Figures 6 through 9 , as a function of $\rho$ from 0 up to 20 , for $\Omega=0.9,1$, 1.1 , and 1.5 , successively. The behavior of the potential versus the distance is consistent with the results of previous works in this domain, using direct integration without approximation [e.g., Buckley,
1968]. The electrostatic response of the plasma is seen as a strongly damped waveform, with a quasi-periodic shape visible only in the imaginary part for $\Omega<1$, and a more or less damped oscillation (the Langmuir wave) in both real and imaginary parts, for $\Omega<1$. Results from the series (18) have been found identical to those obtained from direct numerical integration of (1), except for the case $\Omega=1$, where the singular point makes difficult the numerical integration.

Computation is made using double precision for the coefficients and remains converging up to $\rho \sim 20$, but then requires orders of coefficients larger than $q=50$. This is equivalent to the computation of the sine and cosine functions for large arguments, using their power expansion. It is the reason why the power series expansion (18) is proposed to be used only for small distances, where usual approximations fail.

\section{Comparison With the Landau Wave Approximation}

The Landau wave approximation [Chasseriaux et al., 1972] consists (1) to ignore the contribution of all higher-order poles; (2) to approximate the contribution
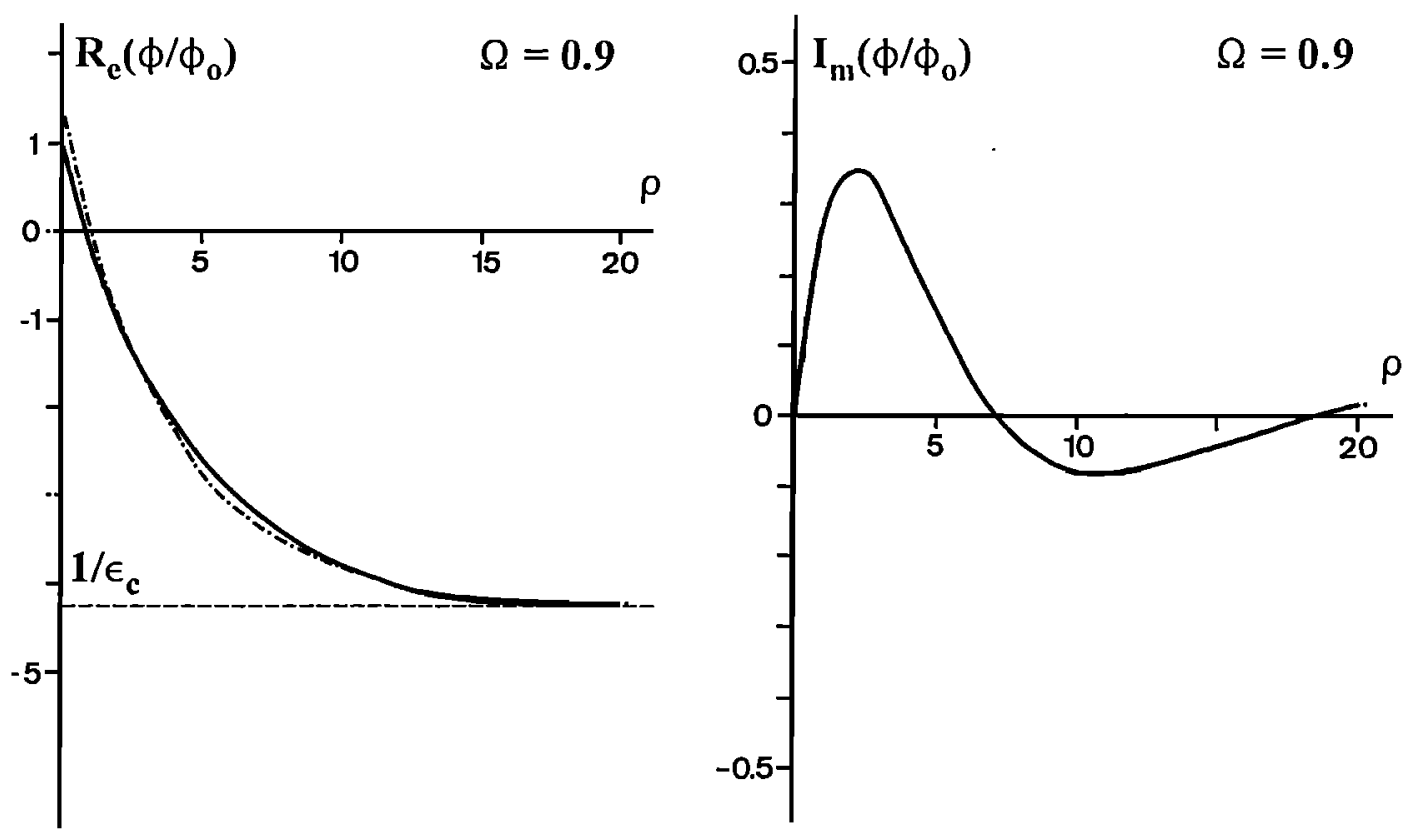

Figure 6. Real and imaginary parts of the normalized potential versus the normalized distance from the source, for $\Omega=0.9$, as obtained using the power expansion (solid line) and the Landau wave approximation (dashed line). Note that the approximation leads to an imaginary part equal to zero for $\mathbf{\Omega} \leq 1$. 


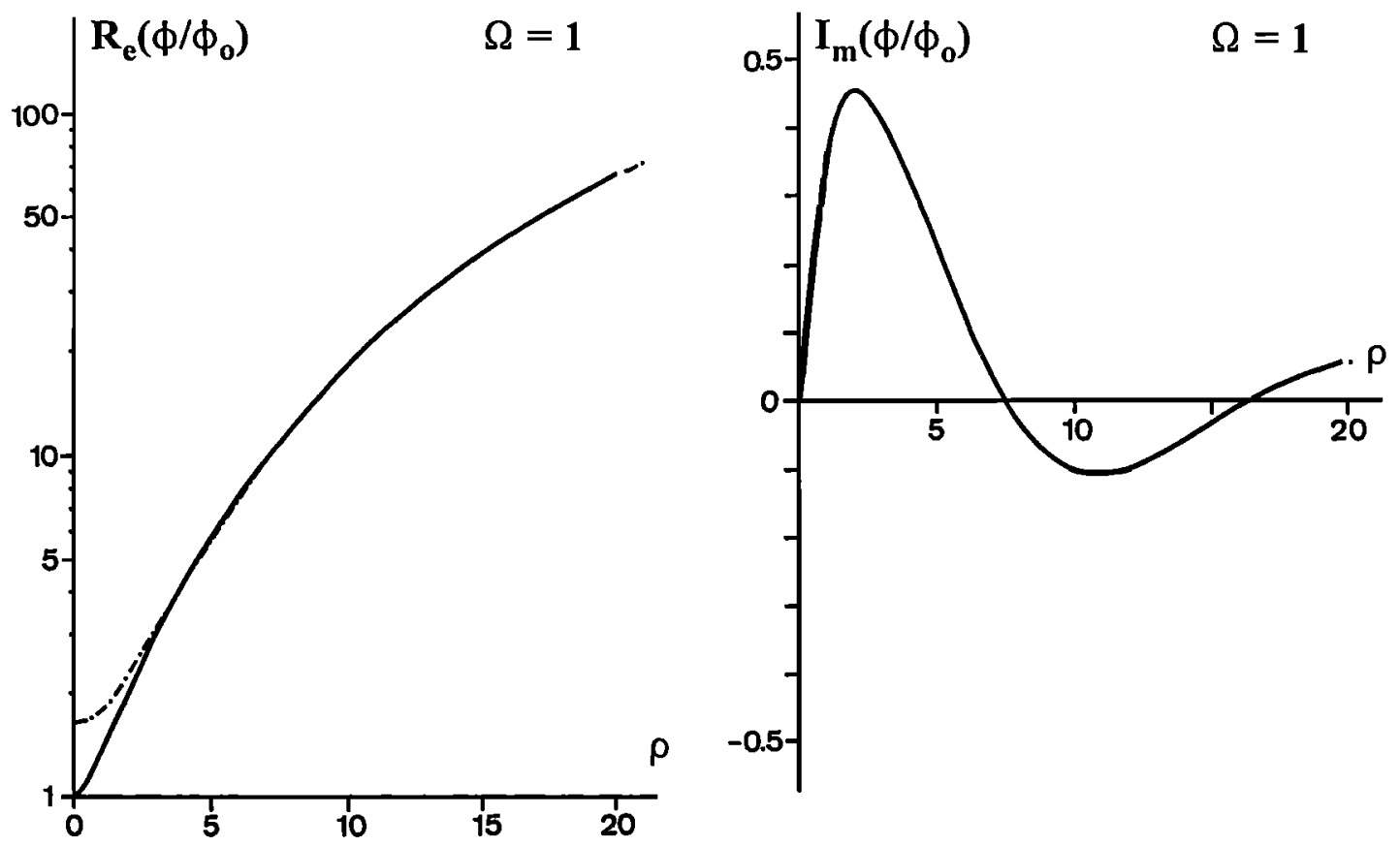

Figure 7. Same as in Figure 6 , for $\Omega=1$.

of the pole $K_{1}$ for $\Omega>1$ by a slowly damped sine wave and that of $K_{-2}$ for $\Omega<1$ to an evanescent wave; and (3) to interpret the resulting potential as due to beating between the cold plasma part $\left(1 / \varepsilon_{c}\right)$ and the

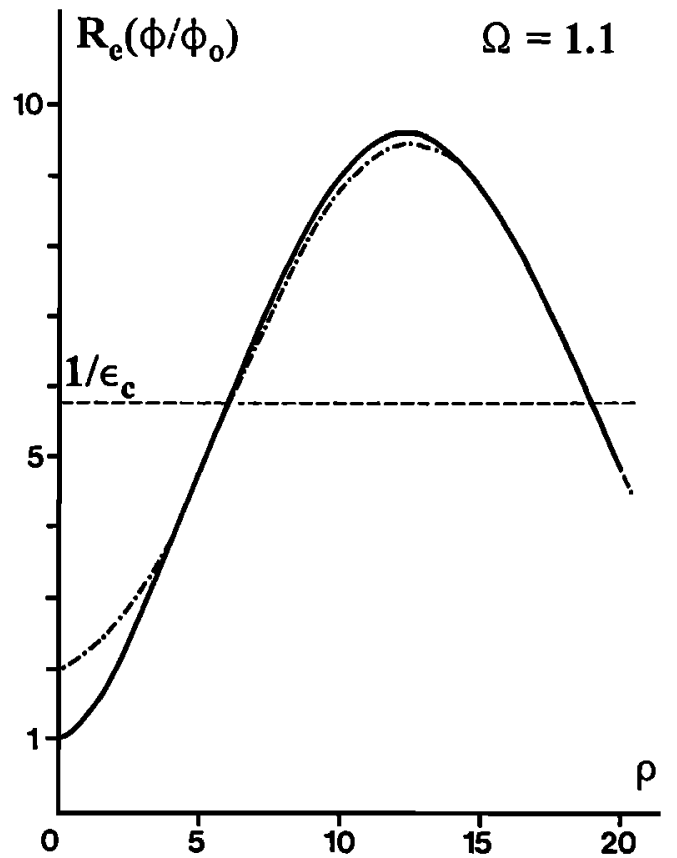

dominating Landau wave. This interpretation needs to be revised, considering that the potential obeys (14), where it could be thought that the cold plasma contribution should be $3 / 4 \varepsilon_{c}$ and the warm

Figure 8. Same as in Figure 6, for $\Omega=1$.1. Here the imaginary part as given by the Landau wave approximation (dashed line) is not zero.

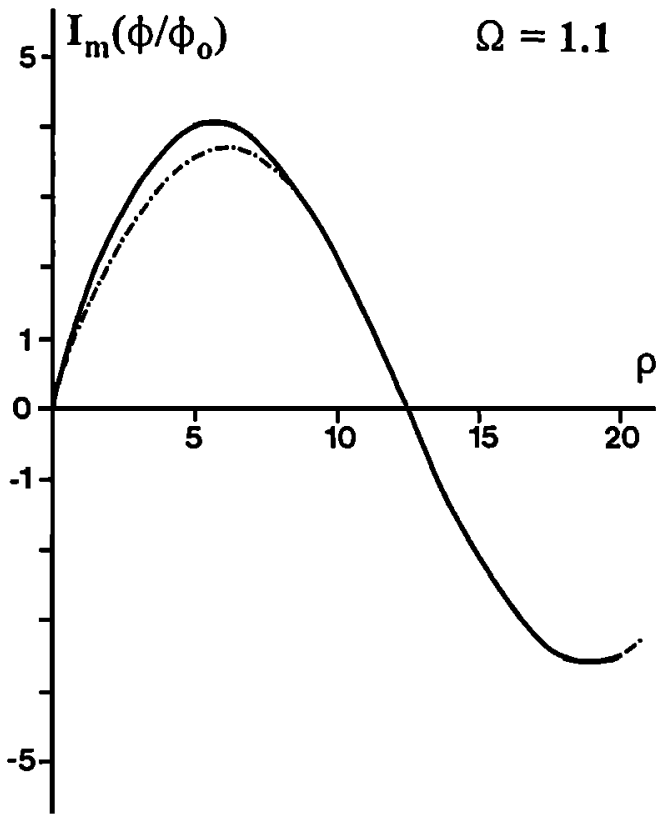



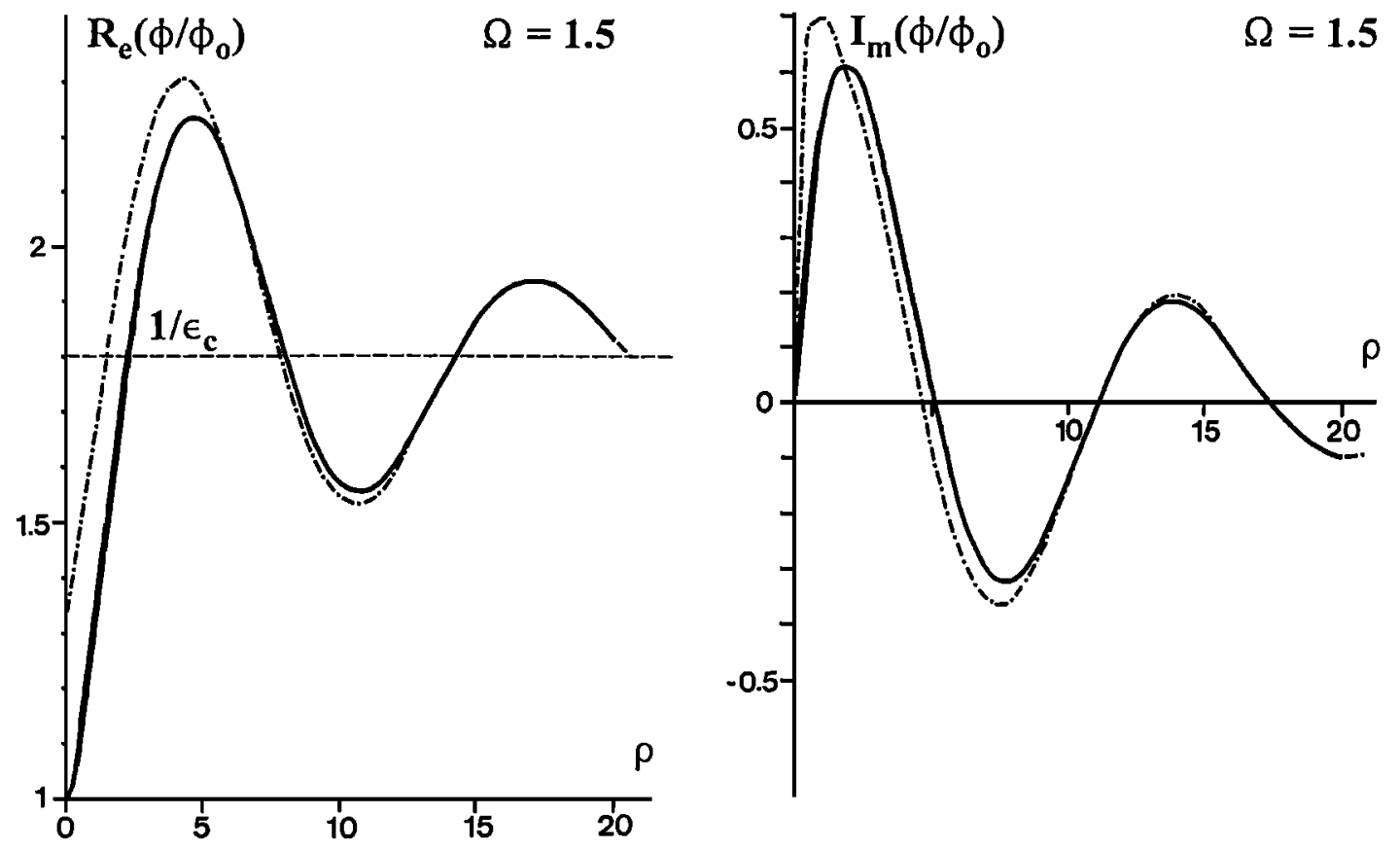

Figure 9. Same as in Figure 8, for $\Omega=1.5$.

contribution should come from the infinite sum of Landau poles. In reality, this assumption is not consistent with the fact that we are considering a Maxwellian thermal distribution independently of the absolute value of the plasma temperature, which could be as low as we want.

Therefore, the correct approach is to evaluate the relative contributions between the dominating Landau poles and the infinite series of higher-order poles. Since these higher-order poles produce highly damped waves, it is generally believed, somewhat incorrectly, that their contribution should be negligible at large distances from the source. In order to evaluate that let us consider $\rho \rightarrow \infty$ in (14). The paradox comes from the fact that for any finite value of $\rho$, there is still an infinity of poles $K_{n}$, such as $\rho K_{n} \rightarrow 0$, the contribution of which could be not negligible since their damping actually remains low. To solve this question, it is necessary to come back to the variable $z_{n}$, the only one for which the Mittag-Leffler expansion is definite. Separating the contributions of the higher-order poles from that of the main ones $\left(K_{1}\right.$ and $\left.K_{-2}\right)$, we rewrite (14)

$$
\begin{gathered}
\frac{\phi}{\phi_{0}}=\frac{3}{4 \varepsilon_{c}}-\frac{2}{\pi} \int_{0}^{\infty} \sum_{n=2}^{|n|-\infty} \frac{b_{n}}{z_{n}+\frac{\rho \Omega}{x \sqrt{2}}} \frac{\sin x d x}{x}+ \\
\frac{2}{\pi} \sum_{n=-2}^{1} \frac{K_{n}^{2}}{1-\Omega^{2}+3 K_{n}^{2}} f\left(K_{n} \rho\right)
\end{gathered}
$$

When $\rho \Omega$ tends to infinity, for a given finite value of $x$, we get from (13)

$$
\sum_{n=2}^{|n|-\infty} \frac{b_{n}}{z_{n}+\frac{p \Omega}{x \sqrt{2}}} \rightarrow-\frac{1}{4 \varepsilon_{c}} \quad\left(\frac{p \Omega}{x \sqrt{2}} \rightarrow \infty\right)
$$

Moreover, since the main contribution to the integral comes from small values of $x$, the more $x$ approaches to zero, the more (31) is valid. Then, after the trivial integration of $\sin x / x$ we obtain the result 
$\frac{\phi}{\phi_{0}} \approx \frac{1}{\varepsilon_{c}}+\frac{2}{\pi} \sum_{n=-2}^{1} \frac{K_{n}^{2}}{1-\Omega^{2}+3 K_{n}^{2}} f\left(K_{n} \rho\right) ;(\rho \Omega \rightarrow \infty)$

which basically validates the so-called Landau wave approximation proposed by Chasseriaux et al. [1972], but with a quite different meaning, since now the contribution of highly damped waves is included for $25 \%$ in the term $1 / \varepsilon_{c}$. Except in the vicinity of $\Omega 21$, where the pole $K_{1}$ lies near the negative real axis, the asymptotic behavior of the function $f\left(K_{n} \rho\right)$, when $\rho$ tends to infinity, is decreasing like $1 / K_{n} \rho$ [Abramowitz and Stegun, 1972]. Thus we see immediately from (32) that the asymptotic value of the potential at large distances is that of the cold plasma, which is visible in Figures 6 through 9. This is consistent with the fact that more the temperature decreases, as $\lambda_{D}$ does, the more the normalized distance $r / \lambda_{D}$ tends to infinity.

The success of the Landau wave approximation for practical applications comes from a larger range of validity than simply seen from its asymptotic behavior. Indeed, for $\Omega_{2} 1$, until $K_{1} p$ lies near the negative real axis, the sum $f\left(K_{1} \rho\right)+f\left(K_{-2} \rho\right)$ reduces to $\pi \exp \left(i K_{1} \rho\right)$, even at relatively small distance [Simonen, 1966]. For $\Omega<1$ the contribution of the imaginary pole $K_{-2}$ is nearly an evanescent wave, which has been proposed by Chasseriaux et al. [1972] to be represented in (32) by the crude approximation $f\left(K_{-2} \rho\right) \sim \pi \exp \left(-\rho\left|K_{-2}\right|\right)$, properly chosen to achieve an analytic continuation through $\Omega=1$. Thus, the conventional Landau wave approximation, used with some success after comparison with experimental data of large-size mutual impedance probes, reads

$$
\frac{\phi}{\phi_{0}} \approx \frac{1}{\varepsilon_{c}}+\frac{2 K_{p}^{2}}{1-\Omega^{2}+3 K_{p}^{2}} \exp \left(i K_{p} p\right) ;(p \Omega \rightarrow \infty)
$$

with $K_{p}=K_{1}$ for $\Omega>1$ and $K_{p}=-K_{-2}$ for $\Omega<1$.

The comparison between the results obtained from the analytic series (18) and the approximation given by (33) is shown in Figures 6 through 9. As a general feature, we see that for the real part of the potential, the Landau wave approximation is quite satisfactory for $\rho$ larger than about 15 , as well as for the imaginary part when $\Omega>1$. But, obviously the approximation is unable to produce the strongly damped waveform of the imaginary part when $\Omega \leq 1$

\section{Application to Mutual Impedance Probes}

Modeling of mutual impedance response is one of the main applications of this work, as the measured response, compared to the theoretical one, is used for plasma diagnostic. We show in Figure 10 an example of the mutual impedance response expected to be obtained from a device developed for the Russian MARS 96 mission. For an electron density of $500 \mathrm{e} / \mathrm{cm}^{3}$ and temperature of $1000 \mathrm{~K}$ in the Martian ionosphere, which are typical values around $300 \mathrm{~km}$ of altitude [Hanson et al., 1977], we get $\lambda_{D} \sim 10 \mathrm{~cm}$. The simplified model of the device is made of two single charges $+Q$ and $-Q$ located $30 \mathrm{~cm}$ each other and a double-point receiving dipole $1-\mathrm{m}$ long (see inset of Figure 10), so that we are everywhere in conditions such as $p<10$. As long as the sensor-receiver system is a high-impedance coupling one, and the source a constant-current generator [Béghin et al., 1982], the induced voltage $V$, in each sensor is the same as the space potential induced by the two charges at this location, and the charge distribution in the source is the same in the plasma as in vacuum. Then, the mutual impedance is defined by

$$
\frac{Z}{Z_{0}}=\frac{\Delta V_{p}}{\Delta V_{0}}
$$

where $\Delta V_{p}$ is the differential voltage between the two receiving sensors $\left(V_{1}-V_{2}\right)$ in the plasma, $\Delta V_{0}$ is its free-space value, and $V_{1}$ or $V_{2}$ are given by

$$
V_{i}=\sum_{j} \operatorname{sign}\left(Q_{j}\right) \frac{\phi_{i}\left(\boldsymbol{\rho}_{i j}\right)}{\rho_{i j}}
$$

where $\rho_{i j}$ is the normalized distance between the charge $Q_{j}$ and the receiving sensor of index $i$.

The modulus and the phase of $Z / Z_{0}$ are plotted in Figure 10 , as calculated using the power expansion (solid line) and the Landau wave approximation (dashed line). The exact solution exhibits a wellmarked maximum for the phase very close to $\Omega=1$, while the modulus reaches a flat maximum around $\Omega \sim 1.5$. The fact that the phase leads to a precise 

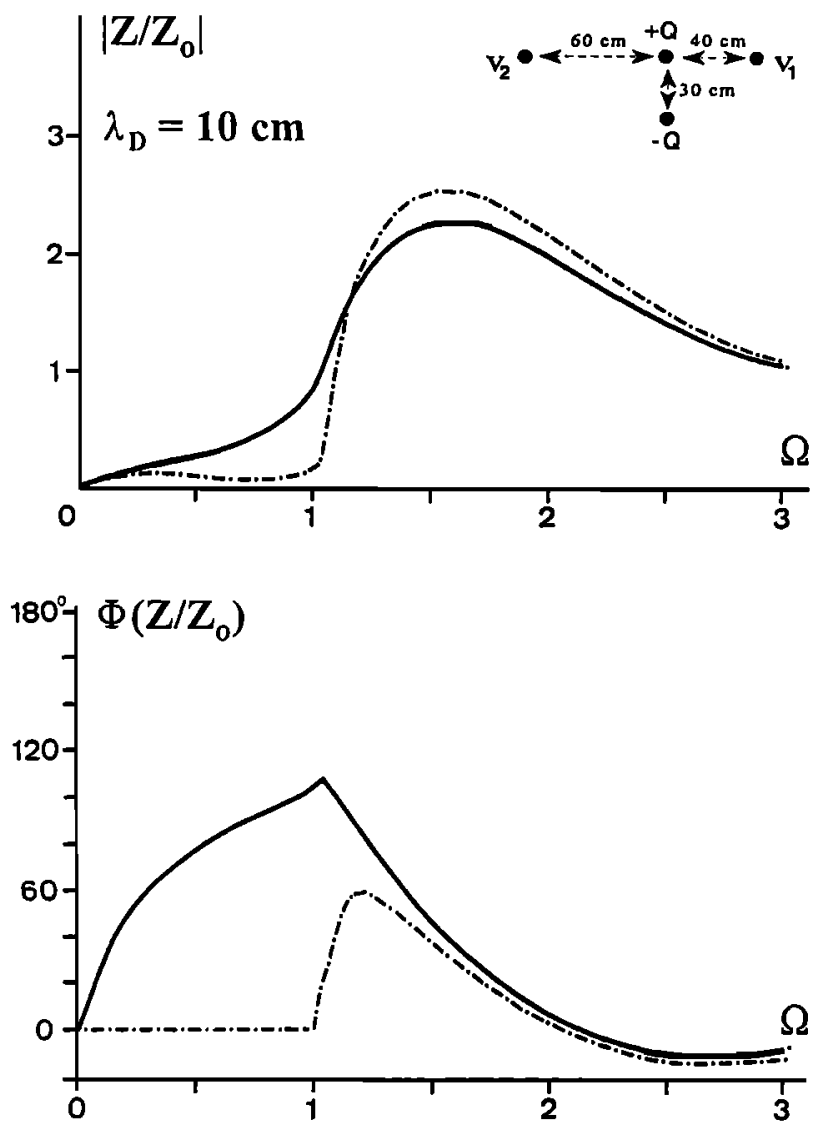

Figure 10. Theoretical normalized response, in modulus and phase, of an idealized mutual impedance probe made of two single charges and two potential sensors, for $\lambda_{D}=10 \mathrm{~cm}$. Shown are the exact value using the power expansion (solid line) and the Landau wave approximation (dashed line).

determination of $\omega_{p}$ is consistent with the measurements performed at large Debye lengths [Décréau et al., 1978]. However, a more realistic modeling would be necessary in the attempt to calculate the true probe response, considering the actual shape and taking account of the charge distribution induced among all conductive surfaces. The method consists of solving (35) on a three-dimensional finite-element grid array, with limit conditions on constant potential surfaces which satisfy the charge neutrality equation. The power series expansion theory is well adapted for such a computation as long as the considered distances are shorter than $\sim 15 \lambda_{D}$. At larger distances the Landau wave approximation would be used.

\section{Conclusion}

A revised mathematical treatment of the classical problem of the electrostatic potential induced by point source in a Maxwellian isotropic plasma has been presented. The new formalism for Mittag-Lefler expansion of the plasma function $1 / \varepsilon_{l}$ leads to a different expression of the potential from that previously considered. The first result is a better understanding of the real contribution of the higher-order Landau poles, which has been found to be included in what was interpreted before as the cold plasma contribution. Then, using the Mittag-Lefler series, the symmetry properties of the plasma dispersion function and the expansion of the auxiliary functions of the family of exponential integrals, the power expansion for the potential has been obtained, with coefficients depending only on the frequency. Moreover, considerations about the asymptotic behavior of this expansion at large distances allows one to justify the classical Landau wave approximation in such conditions. The power series expansion for the potential reveals to be of considerable practical interest for exact computation and probe modeling when sises are smaller than about 15 to $20 \lambda_{D}$.

\section{Appendix}

By integration of the quantity $1 / \varepsilon_{l}-1$ along the negative $K$ axis (where there is no pole), performed using both (6) and (9), we obtain after some development

$$
L_{0}=\frac{\Omega}{\sqrt{2}} \int_{-\infty}^{0} \frac{Z^{\prime}(x) d x}{\Omega^{2}-x^{2} Z^{\prime}(x)}
$$

which is a finite integral except for $\Omega=1$, due to the pole for $x$ infinite. If we extend this integral along a closed contour including the positive imaginary axis $y$, since $Z$ (iy) turns to be purely real, we recover the result given by (26) and (27) for $q=0$, that is, the real part of $L_{0}$ is the residue for the imaginary pole iy $=\Omega /\left(K_{-2} \sqrt{ } 2\right)$ when $\Omega<1$. Using the symmetry properties of $Z$ ', we can also write

$$
\operatorname{Im}\left(L_{0}\right)=-\frac{\Omega}{\sqrt{2}} \operatorname{Im} \int_{0}^{\infty} \frac{Z^{\prime}(x) d x}{\Omega^{2}-x^{2} Z^{\prime}(x)}
$$


For $\Omega \rightarrow 0$ the main contribution of the integrant occurs around $x=0$; then, using the first order expansion of $Z$, we obtain

$$
\operatorname{Im}\left(L_{0}\right) \approx i \sqrt{\frac{\pi}{2}} \frac{\Omega}{2} \quad(\Omega \rightarrow 0)
$$

For $\Omega>1$, expanding the integrant versus $1 / \Omega^{2}$, one obtains the following asymptotic expansion

$$
\begin{aligned}
& \operatorname{Im}\left(L_{0}\right) \approx i \sqrt{\frac{\pi}{2}} \frac{1}{\Omega} \sum_{p=0}^{\infty} \frac{C_{p}}{\Omega^{2 p}} \\
& C_{p}=i \frac{1}{\sqrt{\pi}} \int_{0}^{\infty} x^{2 p}\left[Z^{\prime}(x)\right]^{p+1} d x
\end{aligned}
$$

where the constants $C_{p}$ are computed once and for all. The first ones can be determined analytically after a quite tedious effort using the power expansion of the function $Z^{\prime}$ and the following relation

$$
\sum_{0}^{\infty} \frac{(-1)^{n+1} 2^{2 n+1}(n !)^{2}}{(2 n+1) !}=-\frac{2}{\sqrt{2}} \ln (1+\sqrt{2})
$$

The first three terms of the series $C_{p}$ are

$$
\begin{aligned}
& C_{0}=1 ; \quad C_{1}=\frac{3 \ln (1+\sqrt{2})-\sqrt{2}}{2 \sqrt{2}} \\
& C_{2}=\frac{1}{6}+\frac{31 \ln (1+\sqrt{2})}{18 \sqrt{2}}-\frac{8 \pi}{27}
\end{aligned}
$$

One can notice that the coefficients of the sum in (A4) are close to those of the expansion of the function $\left[\Omega^{2} /\left(\Omega^{2}-1\right)\right]^{c_{1}}$, in accordance with the behavior of $\operatorname{Im}\left(L_{0}\right)$, shown in Figure 5 , as well as when $\Omega$ tends to infinity, as when approaching 1 .

For $0<\Omega<1$ one can find a converging power expansion of $\operatorname{Im}\left(L_{0}\right)$ for the variable $\eta=1 / 2-\Omega^{2}$, with $\eta$ varying from $1 / 2$ to $-1 / 2$, using the fact that $\left|1 / 2-x^{2} Z^{\prime}(x)\right| \geq 1 / 2$. One obtains

$$
\begin{aligned}
& \operatorname{Im}\left(L_{0}\right)=i \Omega \sum_{0}^{\infty}\left[\frac{1}{2}-\Omega^{2}\right]^{p} B_{p} \\
& B_{p}=i \sqrt{2} \operatorname{Im} \int_{0}^{\infty} \frac{Z^{\prime}(x) d x}{\left[\frac{1}{2}-x^{2} Z^{\prime}(x)\right]^{p+1}}
\end{aligned}
$$

where the coefficients $B_{p}$ are constants computed once and for all, as the previous series $C_{p}$. Values of these coefficients for both series are listed in Table Al, up to $p=10$.

Having obtained an analytic expression for $\operatorname{Im}\left(L_{0}\right)$, the problem now is to deduce from it the other coefficients $\operatorname{Im}\left(L_{2 q}\right)$, using a recurrent relationship. Unfortunately, these coefficients do not obey a simple recurrent law do the coefficients $S$. Starting from the definition $L_{0}$ in (17) and using the following relation

$$
\frac{d K_{n}}{d \Omega}=\frac{K_{n}}{\Omega} \frac{1-\Omega^{2}+K_{n}^{2}}{1-\Omega^{2}+3 K_{n}^{2}}
$$

we obtain the differential recurrent equation between the coefficients $L$ and $S$.

$$
\begin{gathered}
3 \Omega^{\frac{p+6}{3}} \frac{d}{d \Omega}\left(L_{p+2} \Omega^{-\frac{p+3}{3}}\right)=\left[p+3-(p+1) \Omega^{2}\right] L_{p}+ \\
\Omega\left(\Omega^{2}-1\right) \frac{d L_{p}}{d \Omega}+S_{p+3}+\left(1-\Omega^{2}\right) S_{p+1}
\end{gathered}
$$

Table A1. First Coefficients of $L_{2 q}$ Series

\begin{tabular}{clr}
\hline$p$ & \multicolumn{1}{c}{$C_{p}$} & \multicolumn{1}{c}{$B_{p}$} \\
\hline 0 & 1. & 0.644588 \\
1 & 0.4348378 & -0.098254 \\
2 & 0.3091567 & 0.120390 \\
3 & 0.2503295 & -0.072666 \\
4 & 0.2151077 & 0.118156 \\
5 & 0.1911903 & -0.079526 \\
6 & 0.1736555 & 0.167482 \\
7 & 0.1601198 & -0.099190 \\
8 & 0.1492772 & 0.289021 \\
9 & 0.1403465 & -0.11875 \\
10 & 0.1328291 & 0.57312 \\
\hline
\end{tabular}


which allows one, in principle, to calculate higher-order terms starting from the power expansions of $L_{0}$. For instance, we obtain for $L_{2}$ the following expressions

$$
\begin{gathered}
\operatorname{Im}\left(L_{2}\right)=-i \sqrt{\frac{\pi}{2}}[\alpha \Omega+\Omega \ln \Omega+ \\
\left.\sum_{p=1}^{\infty} \frac{p+1}{3 p} \frac{C_{p-1}-C_{p}}{\Omega^{2 p-1}}\right] \quad(\Omega>1) \\
\operatorname{Im}\left(L_{2}\right)=i \Omega \sqrt{2}\left[\beta+\frac{1}{3 \sqrt{2}} \sum_{p=1}^{\infty} \frac{B_{p}}{2^{p}}\right. \\
\cdot\left(\frac{\left(1-2 \Omega^{2}\right)^{p}\left[2+p+2 p^{2}\left(\Omega^{2}-1\right)\right]}{2 p(p-1)}+\right. \\
\left.\left.\sum_{k=0}^{p-2} \frac{\left(1-2 \Omega^{2}\right)^{k+1}}{k+1}\right)\right] \quad(\Omega<1)
\end{gathered}
$$

where $\alpha=-0.058053$ and $\beta=-0.4468417$ are integration constants determined numerically from the direct computation of $L_{2}$.

However, for practical reasons, the direct computation of coefficients $L$ for a limited number of frequencies is more efficient than the analytic method, though the number of data to be stored is significantly larger.

\section{References}

Abramowitz, M., and I. A. Stegun, Handbook of Mathematical Functions, Dover, Mineola, N. Y., 1972.

Béghin, C., Excitation de la résonance hybride basse (LHR) par sonde quadripolaire à bord d'une fusée, in Space Research XI, Akademie-Verlag, Berlin, 1071, 1971.

Béghin, C., and R. Debrie, Characteristics of the electric field far from and close to a radiating antenna around the lower hybrid resonance in the ionospheric plasma, J.Plasma Phys., 8, 287, 1972.

Béghin, C., J. F. Karczewski, B. Poirier, R. Debrie, and N. Massevitch, The ARCAD-3 ISOPROBE experiment for high time resolution thermal plasma measurements, Ann. Geophys., 38, 615, 1982.

Buckley, R., Radio frequency properties of a plane grid capacitor immersed in a hot collision-free plasma, $J$. Plasma Phys., 2, 339, 1968.

Chasseriaux J. M., R. Debrie, and C. Renard, Electron density and temperature measurements in the lower ionosphere as deduced from the warm plasma theory of the quadrupole probe, J. Plasma Phys., 8, 231, 1972.

Décréau, P. M. E., C. Béghin, and M. Parrot, Electron density and temperature, as measured by the mutual impedance experiment on board GEOS-1, Space Sci. Rev., 22, 581,1978.

Décréau, P. M. E., S. Perraut, H. de Feraudy, L. Matson, and $O$. Randriamboarison, Electron density and temperature in the cusp and polar cap regions: Contributions from the wave and particle experiment on Viking, in Modeling Magnetospheric Plasma Geophys. Monogr. Ser., vol. 62, edited by G. R. Wilson, p 112, AGU, Washington, D. C., 1991.

Derfler, H., Transients in electron plasmas, in Proc. Int. Conf. Phenom. Ionized Gases 2nd, 282, 1966.

Derfler, H., and T. C. Simonen, Higher-order Landau modes, Phys. Fluids, 12, 269, 1969.

Fried, B. D., and S. D. Conte, The plasma dispersion function, Academic, N. Y., 1961.

Grard, R. J. L., Coupling between two electric' aerials in a warm plasma, Alta Freq., 38, 97, 1969.

Hanson, W. B., S. Sanatini, and D. R. Zuccaro, The Martian ionosphere as observed by the Viking retarding potential analysers, J. Geophys. Res., 82, 4351, 1977.

Jeffreys, H., and B. S. Swirles, Methods of Mathematical Physics, 3rd ed., Cambridge University Press, N. Y., 1966.

Landau, L., On the vibrations of the electron plasma, $J$. Phys. Moscow, 10, 45, 1946.

Navet, M., and P. Bertrand, Multiple "water-bag" model and Landau damping, Phys. Lett., 34A, 117, 1971.

Pottelette, R., M. Hamelin, J. M. Illiano, and B. Lambège, Interpretation of the fine structure of electrostatic waves excited in space, Phys. Fluids, 24, 1517, 1981.

Rooy, B., M. R. Feix, and L. R. O. Storey, Théorie de la sonde quadripolaire en plasma chaud isotrope, Plasma Phys., 14, 275, 1972.

Simonen, T. C., Landau waves, Rep. SUIPR 100 , Inst. for Plasma Res., Stanford Univ., Stanford, Calif., 1966.

Storey, L. R. O., M. P. Aubry, and P. Meyer, A quadripole probe for the study of ionospheric plasma resonances, in Plasma Waves in Space and in the Laboratory, vol. 1 , edited by J. O. Thomas and B. J. Landmark, University Press, Edinburgh, Scotland, p. 303, 1969.

C. Béghin, Laboratoire de Physique et Chimie de l'Environnement, CNRS, 45071 Orléans Cedex, France. (email: span cnesta::beghin or cbeghin@cnrs-orleans.fr)

(Received June 30, 1994; revised November 15, 1994; accepted November 15, 1994.) 